\title{
Stabilized-jellium description of neutral and multiply charged fullerenes $\mathbf{C}_{60}^{x \pm}$
}

\author{
C. Yannouleas and Uzi Landman \\ School of Physics, Georgia Institute of Technology, Atlanta, Georgia 30332-0430
}

(Dated: August 1993; Chem. Phys. Lett. 217, 175 (1994))

\begin{abstract}
A description of neutral and multiply charged fullerenes is proposed based on a stabilized jellium (structureless pseudopotential) approximation for the ionic background and the local density approximation for the $\sigma$ and $\pi$ valence electrons. A recently developed shell-correction method is used to calculate total energies and properties of both the neutral and multiply charged anionic and cationic fullerenes. The effect of the icosahedral symmetry is included perturbatively. The calculated single-particle energy level spectrum of $\mathrm{C}_{60}$ is in good correspondence with experimentally measured ones and previous self-consistent local-density-approximation calculations. For the multiply charged fullerenes, we calculate microscopically the charging energies of $\mathrm{C}_{60}^{x \pm}$ for up to $x=12$ excess charges. A semiclassical interpretation of these results is developed, which views the fullerenes as Coulomb islands possessing a classical capacitance. The calculated values for the first ionization potential and the first electron affinity agree well with the experimental ones. For the second and third ionization potentials, there exist substantial discrepancies in the experimental measurements. Our calculations support the results from charge transfer bracketing experiments and from direct ionization experiments through electron impact. The doubly charged negative ion is found to be a very long-lived metastable species, in agreement with observations.
\end{abstract}

PACS numbers: 


\section{INTRODUCTION}

Charging of macroscopic metal spheres is an old subject with scientific accounts dating back to Coulomb, Faraday, and others [1]. Recently, issues related to electrical charging emerged in connection with quantal nanostructures which are of interest to diverse areas of condensed-matter and atomic, molecular and cluster physics, such as nanofabricated semiconductor devices known as Coulomb islands [2, 3], metal microclusters [4, 5, 6, 7], electron attachment to molecular 8, 9] and alkali-halide [10] clusters, and carbon clusters and fullerenes [11, 12, 13, 14, 15, 16, 17, 18].

We report on a unified theoretical study of the energetics, stability, ionization potentials and electron affinities of neutral and charged carbon fullerenes, $\mathrm{C}_{60}^{x \pm}$, using local density functional theory (LDA) [19] with a stabilized-jellium background [20]. The present approach is an adaptation to the case of fullerenes of a shell-correction method (SCM), developed by us previously for metal microclusters [4, 5]. The main elements of this adaptation consist of employment of the stabilized jellium instead of the usual jellium background, and the use of a generalized electron-density profile (appropriate for description of the fullerene cage). Furthermore, the point-group icosahedral symmetry of the $\mathrm{C}_{60}$ cage is introduced via a perturbative treatment.

The electronic structure of fullerenes has been investigated extensively using various methods ranging from $a b$ initio all-electron quantum chemical Hartree-Fock calculations, and self-consistent Kohn-Sham LDA calculations in conjunction with nonlocal pseudopotentials or a jellium background for the ions, to simplified free-electron [21, 22] and particle-ona-sphere models [23]. Our generalized shell-correction method (GSCM) which combines elements of the LDA methodology with simplifications circumventing self-consistent solution of the Kohn-Sham (KS) equations represents a substantial improvement over certain previous simplified methods, yielding results in quantitative agreement with self-consistent KS-LDA $a b$ initio pseudopotential calculations (see, e.g., [24, 25, 26]), and experimental data.

Additionally, our method permits us to investigate, within the local density approximation, the important class of multiply anionic fullerene systems, where the familiar KS-LDA is known to fail [4, 5]. Until now, in all cases but one [27], properties of anionic fullerenes (whose energetics is relevant not only for understanding the properties of free molecules, but also those of fullerite intercalation compounds [29] and complexes [30]) have been treated 
within the Hartree-Fock approximation [16, 30, 31] or estimated with a simplified classical electrostatic model [22, 29], associated with the particle-on-a-sphere model. Both these methods, however, omit essential theoretical ingredients, namely the correlation energy in the case of the former, and the shell structure and appropriate radial and angular density distribution of the electronic charge in the case of the latter. The recent study by Pederson and Quong [27], using an LDA all-electron full-potential Gaussian-orbital basis, was performed within the ansatz of a localized basis expansion of the Kohn-Sham orbitals, yielding results in good agreement with those obtained by us using the GSCM.

\section{LDA THEORETICAL METHOD}

\section{Stabilized jellium approximation}

Fullerenes and related carbon structures have been extensively investigated using ab initio local-density-functional methods and self-consistent solutions of the Kohn-Sham (KS) equations [24, 32]. For metal clusters, replacing the ionic cores with a uniform jellium background was found to describe well their properties within the KS-LDA method (see references in Refs. [4, 5]). Motivated by these results, several attempts to apply the jellium model in conjunction with LDA to investigations of fullerenes have appeared recently [33, 34, 35]. Our approach differs from the earlier ones in several aspects and, in particular, in the adaptation to the case of finite systems of the stabilized-jellium (or structureless pseudopotential) energy density functional (see eq. (11) below and Ref. [20]).[45]

An important shortcoming of the standard jellium approximation for fullerenes (and other systems with high density, i.e., small $r_{s}$ ) results from a well-known property of the jellium at high electronic densities, namely that the jellium is unstable and yields negative surfaceenergy contribution to the total energy [20], as well as unreliable values for the total energy. These inadequacies of the standard jellium model can be rectified by pseudopotential corrections. A modified-jellium approach which incorporates such pseudopotential corrections and is particularly suited for our purposes here, is the structureless pseudopotential model or stabilized jellium approximation developed in Ref. [20].

In the stabilized jellium, the total energy $E_{\text {pseudo, }}$, as a functional of the electron density 
$\rho(\mathbf{r})$, is given by the expression

$$
E_{\text {pseudo }}\left[\rho, \rho_{+}\right]=E_{\text {jell }}\left[\rho, \rho_{+}\right]+\langle\delta v\rangle_{W S} \int \rho(\mathbf{r}) \mathcal{U}(\mathbf{r}) d \mathbf{r}-\widetilde{\varepsilon} \int \rho_{+}(\mathbf{r}) d \mathbf{r}
$$

where by definition the function $\mathcal{U}(\mathbf{r})$ equals unity inside, but vanishes, outside the jellium volume. $\rho_{+}$is the density of the positive jellium background (which for the case of $\mathrm{C}_{60}$ is taken as a spherical shell, of a certain width $2 d$, centered at 6.7 a.u. ). $E_{\text {pseudo }}$ in eq. (1) is the standard jellium-model total energy, $E_{\text {jell }}$, modified by two corrections. The first correction adds the effect of an average (i.e., averaged over the volume of a WignerSeitz cell) difference potential, $\langle\delta v\rangle_{W S} \mathcal{U}(\mathbf{r})$, which acts on the electrons in addition to the standard jellium attraction and is due to the atomic pseudopotentials (in this work, we use the Ashcroft empty-core pseudopotential, specified by a core radius $r_{c}$, as in Ref. [20]). The second correction subtracts from the jellium energy functional the spurious electrostatic self-repulsion of the positive background within each cell; this term makes no contribution to the effective electronic potential.

Following Ref. [20], the bulk stability condition (eq. (25) in Ref. [20]) determines the value of the pseudopotential core radius $r_{c}$, as a function of the bulk Wigner-Seitz radius $r_{s}$. Consequently, the difference potential can be expressed solely as a function of $r_{s}$ as follows (energies in $R y$, distances in a.u.):

$$
\langle\delta v\rangle_{W S}=-\frac{2}{5}\left(\frac{9 \pi}{4}\right)^{2 / 3} r_{s}^{-2}+\frac{1}{2 \pi}\left(\frac{9 \pi}{4}\right)^{1 / 3} r_{s}^{-1}+\frac{1}{3} r_{s} \frac{d \varepsilon_{c}}{d r_{s}}
$$

where $\varepsilon_{c}$ is the per particle electron-gas correlation energy (in our calculation, we use the Gunnarsson-Lundqvist exchange and correlation energy functionals (see Refs. [4, 5])).

The electrostatic self-energy, $\widetilde{\varepsilon}$, per unit charge of the uniform positive jellium is given by

$$
\widetilde{\varepsilon}=6 v^{2 / 3} / 5 r_{s}
$$

where $v$ is the valence of the atoms ( $v=4$ for carbon).

\section{Shell-correction method}

Besides the familiar KS-LDA approach, an alternative LDA method, which has been used in studies of metal clusters [4, 5, 36], is based on an extended Thomas Fermi (ETF) variational procedure using a parametrized density profile $\rho\left(\mathbf{r} ;\left\{\gamma_{i}\right\}\right)$, with $\left\{\gamma_{i}\right\}$ as variational 
parameters [5]. In the case of metal clusters, the energy density functional that is variationally minimized consists of a kinetic-energy functional $T[\rho]$, including terms up to 4th order in the density gradients (see Ref. [5]), and of potential terms according to the standard jellium-LDA functional. The effective single-particle potentials and associated single-particle energy spectra obtained by this method for metal clusters provide a good approximation to the corresponding ones obtained from KS-LDA calculations [4, 5], and have been extensively used [36] in studies of the optical properties of metal clusters. To apply the ETF-LDA method to carbon fullerenes, we generalize it by employing potential terms according to the stabilized-jellium functional (1).

Another required generalization consists in employing a parametrized electron-density profile that accounts for the hollow cage-structure of the fullerenes. Such a density profile is provided by the following adaptation of a generalization of an inverse Thomas-Fermi distribution, used earlier in the context of nuclear physics [37], i.e.,

$$
\rho(r)=\rho_{0}\left(\frac{F_{i, o} \sinh \left[w_{i, o} / \alpha_{i, o}\right]}{\cosh \left[w_{i, o} / \alpha_{i, o}\right]+\cosh \left[(r-R) / \alpha_{i, o}\right]}\right)^{\gamma_{i, o}}
$$

where $R=6.7$ a.u. is the radius of the fullerene cage. $w, \alpha$, and $\gamma$ are variables to be determined by the ETF-LDA minimization. For $R=0$ and large values of $w / \alpha$, expression (4) approaches the more familiar inverse Thomas-Fermi distribution, with $w$ the width, $\alpha$ the diffuseness and $\gamma$ the asymmetry of the profile around $r=w$. There are a total of six parameters to be determined, since the indices $(i, o)$ stand for the regions inside $(r<R)$ and outside $(r>R)$ the fullerene cage. $F_{i, o}=\left(\cosh \left[w_{i, o} / \alpha_{i, o}\right]+1\right) / \sinh \left[w_{i, o} / \alpha_{i, o}\right]$ is a constant guaranteeing that the two parts of the curve join smoothly at $r=R$. The density (44) peaks at $r=R$ and then falls towards smaller values both inside and outside the cage (see top panel of Fig. 1).

However, apart from the effective potentials, other properties of metal clusters - and by analogy of fullerenes - determined via the ETF-LDA method, such as ionization potentials (IPs), electron affinities (EAs), and total energies, compare with the self-consistently calculated KS-LDA values only in an average sense, i.e., they do not exhibit shell effects. In our present study, shell effects are incorporated in the GSCM according to the procedure of Refs. [4, 5]. As elaborated in Ref. [5], shell effects in the total energy are contained, to first order in $\delta \rho_{\mathrm{KS}}\left(\delta \rho_{\mathrm{KS}}=\rho_{\mathrm{KS}}-\widetilde{\rho}\right.$, where $\widetilde{\rho}$ is the ETF-LDA optimized density specified above and $\rho_{\mathrm{KS}}$ is the self-consistent $\mathrm{KS}$ density), in the sum $\sum_{i} \widetilde{\varepsilon}_{i}$, where $\widetilde{\varepsilon}_{i}$ are the single-particle energies 


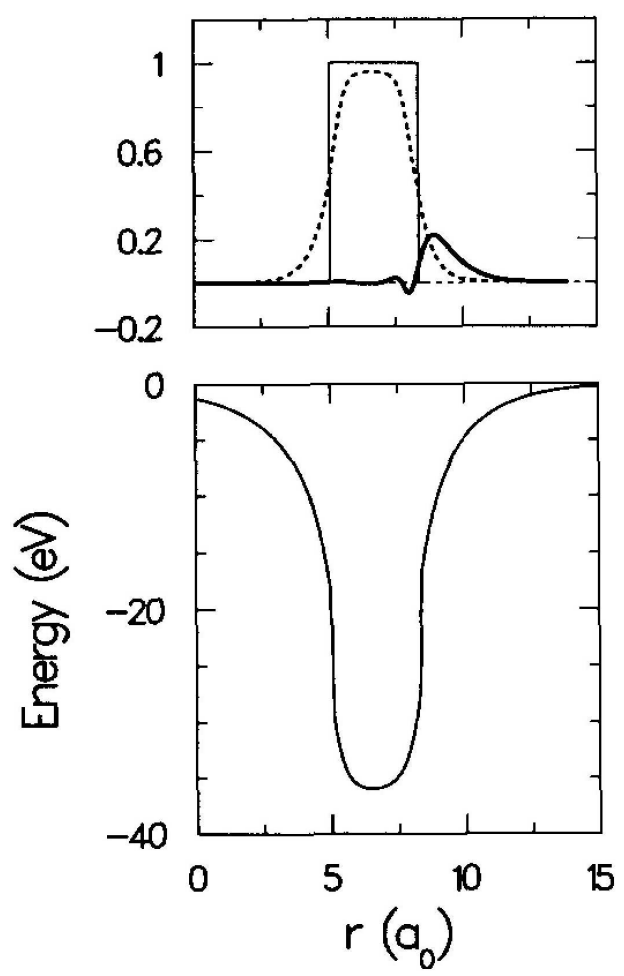

FIG. 1: Bottom panel: The stabilized-jellium LDA potential obtained by the ETF method for the neutral $\mathrm{C}_{60}$ molecule. The Wigner-Seitz radius for the jellium bacground is 1.23 a.u. Note the asymmetry of the potential about the minimum. The associated difference potential $\langle\delta v\rangle_{W S}=-9.61 \mathrm{eV}$.

Top panel: Solid line: Radial density of the positive jellium background. Dashed line: ETF electronic density. Note its asymmetry about the maximum. Thick solid line: The difference (multiplied by 10) of electronic ETF densities between $\mathrm{C}_{60}^{5-}$ and $\mathrm{C}_{60}$. It illustrates that the excess charge accumulates in the outer perimeter of the total electronic density. All densities are normalized to the density of the positive jellium background.

of the ETF effective potential, and the sum extends over the occupied levels. Consequently, we replace the kinetic-energy $\widetilde{T}$ in the ETF-LDA functional by

$$
T_{\mathrm{sh}}=\sum_{i} \widetilde{\varepsilon}_{i}-\int \widetilde{\rho}(\mathbf{r}) \widetilde{V}(\mathbf{r} ; \widetilde{\rho}(\mathbf{r})) d \mathbf{r}
$$

where $\widetilde{V}(\mathbf{r} ; \widetilde{\rho}(\mathbf{r}))$ is the effective potential produced by the ETF method [4, 5] (as an example, see lower panel Fig. 1 for the ETF potential associated with the neutral $\mathrm{C}_{60}$ ). As a result, the total energy, $E_{\mathrm{sh}}$, including the shell correction, $\Delta E_{\mathrm{sh}}=T_{\mathrm{sh}}-\widetilde{T}$, is given by $E_{\mathrm{sh}}[\widetilde{\rho}]=$ 
$T_{\text {sh }}-\widetilde{T}+\widetilde{E}[\widetilde{\rho}]$, where $\widetilde{E}$ is the ETF-LDA energy-density functional.

After some rearrangenments, the shell-corrected total energy $E_{\mathrm{sh}}[\widetilde{\rho}]$ in the GSCM can be written in functional form as follows

$$
E_{\mathrm{sh}}[\widetilde{\rho}]=\sum_{i} \widetilde{\varepsilon}_{i}-\int\left\{\frac{1}{2} \widetilde{V}_{H}(\mathbf{r})+\widetilde{V}_{\mathrm{xc}}(\mathbf{r})\right\} \widetilde{\rho}(\mathbf{r}) d \mathbf{r}+\int \widetilde{\mathcal{E}}_{\mathrm{xc}}[\widetilde{\rho}(\mathbf{r})] d \mathbf{r}+E_{I}-\widetilde{\varepsilon} \int \rho_{+}(\mathbf{r}) d \mathbf{r},
$$

where $\widetilde{V}_{H}$ and $\widetilde{V}_{\text {xc }}$ are the Hartree and exchange-correlation electronic potentials, $\widetilde{\mathcal{E}}_{\text {xc }}$ is the exchange-correlation energy density functional, and $E_{I}$ is the energy of the positive jellium background. The specific way of writing the functional $E_{\mathrm{sh}}$ above was chosen so that its similarity in form to the Harris functional [38] is evident. We note that our method differs from that approach in that the optimization of the input density is achieved by us through a variational ETF method, which does not require a step-by-step matrix diagonalization. While our focus in the previous papers [4, 5] was on jellium models for metal clusters, the very good agreement between our results and those obtained via self-consistent KS-LDA jellium calculations [4, 5] suggested that it would be worthwhile to explore the application of our SCM to more general electronic systems extending beyond metal clusters. The present study is an example of such an application of the SCM to fullerenes.

\section{Icosahedral splitting}

Heretofore, the point-group icosahedral symmetry of $\mathrm{C}_{60}$ was not considered, since the molecule was treated as a spherically symmetric cage. This is a reasonable zeroth-order approximation as noticed by several authors [22, 24, 35, 39]. However, considerable improvement is achieved when the effects of the point-group icosahedral symmetry are considered as a next-order correction (mainly the lifting of the angular momentum degeneracies).

The method of introducing the icosahedral splittings is that of the crystal field theory. Thus, we will use the fact that the bare electrostatic potential from the ionic cores, considered as point charges, acting upon an electron, obeys the well-known expansion theorem [40]

$$
U(\mathbf{r})=-v e^{2} \sum_{i} \frac{1}{\left|\mathbf{r}-\mathbf{r}_{i}\right|}=-\sum_{l=0}^{\infty} \sum_{m=-l}^{l} \kappa_{l}(r) C_{l}^{m} Y_{l}^{m}(\theta, \phi),
$$

where the angular coefficients $C_{l}^{m}$ are given through the angular coordinates $\theta_{i}, \phi_{i}$ of the carbon atomic cores, namely,

$$
C_{l}^{m}=\sum_{i} Y_{l}^{m *}\left(\theta_{i}, \phi_{i}\right)
$$




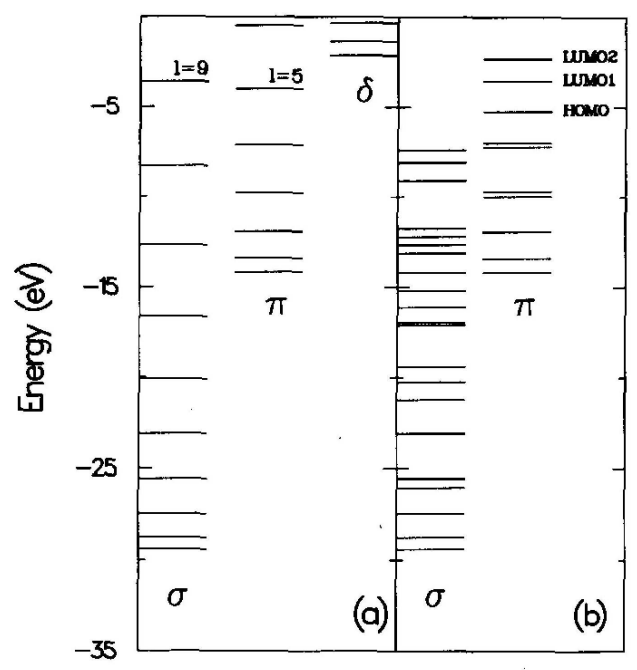

FIG. 2: (a) The single-particle levels of the ETF-LDA potential for $\mathrm{C}_{60}$ shown in Fig. 1. Because of the spherical symmetry, they are characterized by the two principle quantum numbers $n_{r}$ and $l$, where $n_{r}$ is the number of radial nodes and $l$ the angular momentum. They are grouped in three bands labeled $\sigma\left(n_{r}=0\right), \pi\left(n_{r}=1\right)$, and $\delta\left(n_{r}=2\right)$. Each band starts with an $l=0$ level.

(b) The single-particle levels for $\mathrm{C}_{60}$ after the icosahedral splittings are added to the spectra of (a). The tenfold degenerate HOMO $\left(\mathrm{h}_{u}\right)$ and the sixfold degenerate LUMO1 $\left(\mathrm{t}_{1 u}\right)$ and LUMO2 $\left(\mathrm{t}_{1 g}\right)$ are denoted; they originate from the spherical $l=5$ and $l=6\left(\mathrm{t}_{1 g}\right) \pi$ levels displayed in panel (a). For the $\sigma$ electrons, the icosahedral perturbation strongly splits the $l=9$ level of panel (a). There result five sublevels which straddle the $\sigma$-electron gap as follows: two of them (the eightfold degenerate $g_{u}$, and the tenfold degenerate $h_{u}$ ) move down and are fully occupied resulting in a shell closure (180 $\sigma$ electrons in total). The remaining unoccupied levels, originating from the $l=9 \sigma$ level, are sharply shifted upwards and acquire positive values.

where $*$ denotes complex conjugation.

We take the radial parameters $\kappa_{l}(r)$ as constants, and determine their value by adjusting the icosahedral single-particle spectra $\varepsilon_{i}^{\text {ico }}$ to reproduce the pseudopotential calculation of Ref. [24], which are in good agreement with experimental data. Our spectra without and with icosahedral splitting are shown in Fig. 2a and Fig. 2b, respectively. We find that a close reproduction of the results of ab initio LDA calculations [24, 25, 26] is achieved when the Wigner-Seitz radius for the jellium background is 1.23 a.u. The shell corrections including the icosahedral splittings are calculated using the icosahedral single-particle energies $\varepsilon_{i}^{\text {ico }}$ in 
eq. (5). The average quantities $(\widetilde{\rho}$ and $\widetilde{V})$ are maintained as those specified through the ETF variation with the spherically symmetric profile of eq. (4). This is because the first-order correction to the total energy (resulting from the icosahedral perturbation) vanishes, since the integral over the sphere of a spherical harmonic $Y_{l}^{m}(l>0)$ vanishes.

\section{RESULTS}

\section{Ionization potentials and electron affinities}

Having specified the appropriate Wigner-Seitz radius $r_{s}$ and the parameters $\kappa_{l}$ of the icosahedral crystal field through a comparison with the pseudopotential LDA calculations for the neutral $\mathrm{C}_{60}$, we calculate the total energies of the cationic and anionic species by allowing for a change in the total electronic charge, namely by imposing the constraint

$$
4 \pi \int \rho(r) r^{2} d r=240 \pm x
$$

where $\rho(r)$ is given by eq. (44). The shell-corrected and icosahedrally perturbed first and higher ionization potentials $I_{x}^{\text {ico }}$ are defined as the difference of the ground-state shellcorrected total energies $E_{\mathrm{sh}}^{\mathrm{ico}}$ as follows:

$$
I_{x}^{\mathrm{ico}}=E_{\mathrm{sh}}^{\mathrm{ico}}\left(N_{e}=240-x ; Z=240\right)-E_{\mathrm{sh}}^{\mathrm{ico}}\left(N_{e}=240-x+1 ; Z=240\right),
$$

where $N_{e}$ is the number of electrons in the system and $x$ is the number of excess charges on the fullerenes (for the excess charge, we will find convenient to use two different notations $x$ and $z$ related as $x=|z|$. A negative value of $z$ corresponds to positive excess charges). $Z=240$ denotes the total positive charge of the jellium background.

The shell-corrected and icosahedrally perturbed first and higher electron affinities $A_{x}^{\text {ico }}$ are similarly defined as

$$
A_{x}^{\mathrm{ico}}=E_{\mathrm{sh}}^{\mathrm{ico}}\left(N_{e}=240+x-1 ; Z=240\right)-E_{\mathrm{sh}}^{\mathrm{ico}}\left(N_{e}=240+x ; Z=240\right) .
$$

We have also calculated the corresponding average quantities $\widetilde{I}_{x}$ and $\widetilde{A}_{x}$, which result from the ETF variation with spherical symmetry (that is without shell and icosahedral symmetry corrections). Their definition is the same as in Eqs. (10) and (11), but with the index sh replaced by a tilde and the removal of the index $i c o$. 
TABLE I: ETF (spherically averaged, denoted by a tilde) and shell-corrected (denoted by a superscript $i$ co to indicate that the icosahedral splittings of energy levels have been included). IPs and EAs of fullerenes $\mathrm{C}_{60}^{x \pm}$. Energies in $\mathrm{eV} . r_{s}=1.23$ a.u.

\begin{tabular}{ccccc}
$x$ & $\widetilde{I}_{x}$ & $I_{x}^{\text {ico }}$ & $\widetilde{A}_{x}$ & $A_{x}^{\text {ico }}$ \\
\hline 1 & 5.00 & 7.40 & 2.05 & 2.75 \\
2 & 7.98 & 10.31 & -0.86 & -0.09 \\
3 & 10.99 & 13.28 & -3.75 & -2.92 \\
4 & 14.03 & 16.25 & -6.60 & -5.70 \\
5 & 17.09 & 19.22 & -9.41 & -8.41 \\
6 & 20.18 & 22.20 & -12.19 & -11.06 \\
7 & 23.29 & 25.24 & -14.94 & -14.85 \\
8 & 26.42 & 28.31 & -17.64 & -17.24 \\
9 & 29.57 & 31.30 & -20.31 & -19.49 \\
10 & 32.73 & 34.39 & -22.94 & -21.39 \\
11 & 35.92 & 39.36 & -25.53 & -22.93 \\
12 & 39.12 & 42.51 & -28.07 & -23.85
\end{tabular}

In our calculations of the charged fullerene molecule, the $r_{s}$ value and the icosahedral splitting parameters $\left(\kappa_{l}\right.$, see Eq. (7), and discussion below it) were taken as those which were determined by our calculations of the neutral molecule, discussed in the previous section. The parameters which specify the ETF electronic density (Eq. (44)) are optimized for the charged molecule, thus allowing for relaxation effects due to the excess charge. This procedure is motivated by results of previous electronic structure calculations for $\mathrm{C}_{60}^{+}$and $\mathrm{C}_{60}^{-}$[25, 26], which showed that the icosahedral spectrum of the neutral $\mathrm{C}_{60}$ shifts almost rigidly upon charging of the molecule.

Shell-corrected and ETF calculated values of ionization potentials (IPs) and electron affinities (EAs), for values of the excess charge up to 12 units, are summarized in Table I (for $r_{s}=1.23$ a.u.). Our shell-corrected results for the first electron affinity and first ionization potential are in good agreement with the experimental values $(2.75 \mathrm{eV}$ [41] and $7.54 \mathrm{eV}$ [42], respectively). For the second affinity, we find a small negative value of -0.09 $\mathrm{eV}$ indicating that $\mathrm{C}_{60}^{2-}$ is a metastable species (for lifetime estimates, see below). For the 
second ionization potential our value of $10.31 \mathrm{eV}$ is close to that determined experimentally by McElvany et al. (9.7 eV) [11] using charge-transfer bracketing experiments, and most recently by Sai Baba et al. $(10.3 \mathrm{eV})$ [12] using electron impact ionization. There is a controversy about this value, since several other experiments have found values that vary from $8.5 \mathrm{eV}$ to $12.25 \mathrm{eV}$ (for a detailed review of related experimental measurements available in the literature, cf. Ref. [12], and references therein). Our theoretical calculations support the measurements of McElvany et al. and Sai Baba et al., epecially since another quantity namely, the appearance energy of $\mathrm{C}_{60}^{3+}$, that is, the sum of $\sum_{x=1}^{3} I_{x}^{\text {ico }}$ - which was measured in the recent work of Ref. [12] is in very good agreement with the value calculated by us. Indeed, the appearance energy of $\mathrm{C}_{60}^{3+}$ determined in Ref. [12] by linear extrapolation of the ionization efficiency curves was found to be $33.2 \pm 1 \mathrm{eV}$. This value should be considered as the upper limit, due to a curvature at the foot of the ionization efficiency curve. Ref. [12] estimates that this curvature leads to an overestimation of $2 \mathrm{eV}$. Taking this correction into account, the resulting experimental value of $31.2 \pm 1 \mathrm{eV}$ is in close agreement with our theoretical value of $30.99 \mathrm{eV}$.

The IPs and EAs calculated by us are plotted against the excess charge $x$ in Fig. 3. For the ETF results (see Fig. 3a) the dependence is linear to a remarkable degree. Small deviations from linearity, however, are discernible and are due to the variations of electronic spill-out with varying excess charge. An inspection of the shell-corrected results in Fig. 3b reveals that they also exhibit a linear relationship with $x$ within each electronic shell. Discontinuities due to shell openings are clearly discernible $[1 \leq x \leq 10$ for IPs corresponds to removal of electrons from the same $\mathrm{h}_{u}$ shell, while $11 \leq x \leq 12$ corresponds to removal of an electron from the shell immediately below it ( $\mathrm{g}_{g}$ shell); $1 \leq x \leq 6$ for the EAs corresponds to adding an electron to the same $t_{1 u}$ shell, while $7 \leq x \leq 12$ corresponds to an extra electron in the same $\mathrm{t}_{1 g}$ shell. The gap between the $\mathrm{t}_{1 u}$ and the $\mathrm{t}_{1 \mathrm{~g}}$ shell is approximately $1.23 \mathrm{eV}$, while the gap between the $\mathrm{h}_{u}$ shell and the $\mathrm{g}_{g}$ shell is $\left.1.75 \mathrm{eV}\right]$. The bending of the shell-corrected affinities around $x=9-12$ is due to the fact that the electron emission for such highly charged states of the free molecule is barrierless and thus the uncertainty of the LUMO energy is high (see below). In the case of intercalated or endohedral compounds, the LUMO is drastically more bounded, due to the attraction of the ionic intercalant, and one should expect a strong linear relation even for this range of excess charge. 


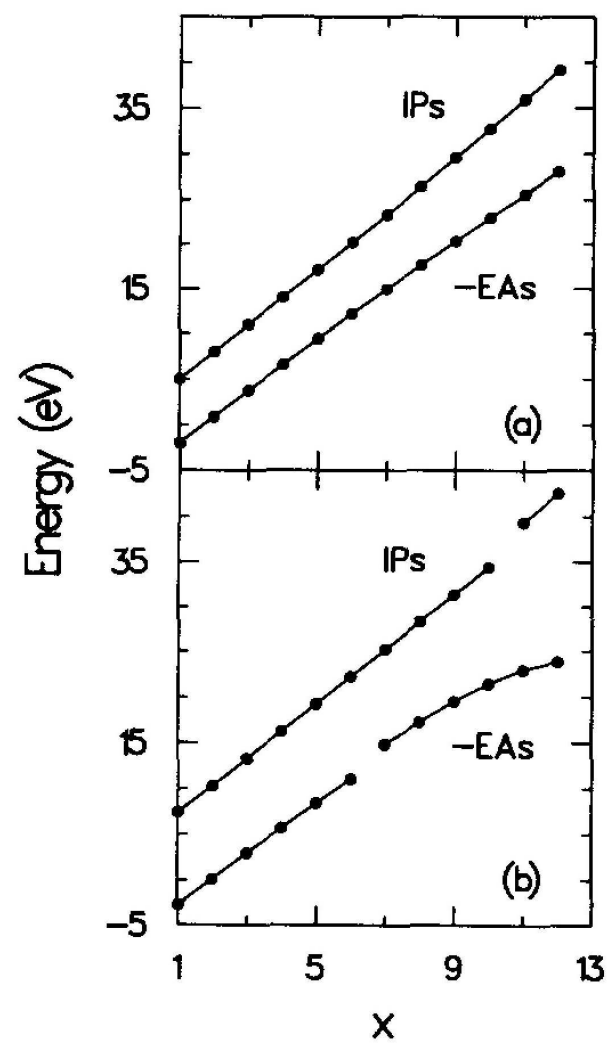

FIG. 3: (a) Extended Thomas-Fermi LDA Ionization potentials $\left(\widetilde{I}_{x}\right)$ and electron affinities $\left(-\widetilde{A}_{x}\right)$ as a function of the excess charge $x$.

(b) Shell-corrected icosahedral IPs $\left(I_{x}^{\text {ico }}\right)$ and - EAs $\left(-A_{x}^{\text {ico }}\right)$ as a function of the excess charge $x$.

\section{Charging energies and capacitance}

Fig. 4a shows that the variation of the total ETF-LDA energy difference (appearance energies) $\Delta \widetilde{E}(z)=\widetilde{E}(z)-\widetilde{E}(0)$, as a function of excess charge $z(|z|=x)$, exhibits a parabolic behavior. The inset in Fig. 4a exhibiting the quantity

$$
\widetilde{g}(z)=\frac{\widetilde{E}(z)-\widetilde{E}(0)}{z}+\widetilde{A}_{1},
$$

plotted versus $z$ (open squares), shows a straight line which crosses the zero energy line at $z=1$. As a result the total ETF-LDA energy has the form,

$$
\widetilde{E}(z)=\widetilde{E}(0)+\frac{z(z-1) e^{2}}{2 C}-\widetilde{A}_{1} z
$$

Equation (13) indicates that fullerenes behave on the average like a capacitor having a capacitance $C$ (the second term on the rhs of eq. (13) corresponds to the charging energy of 

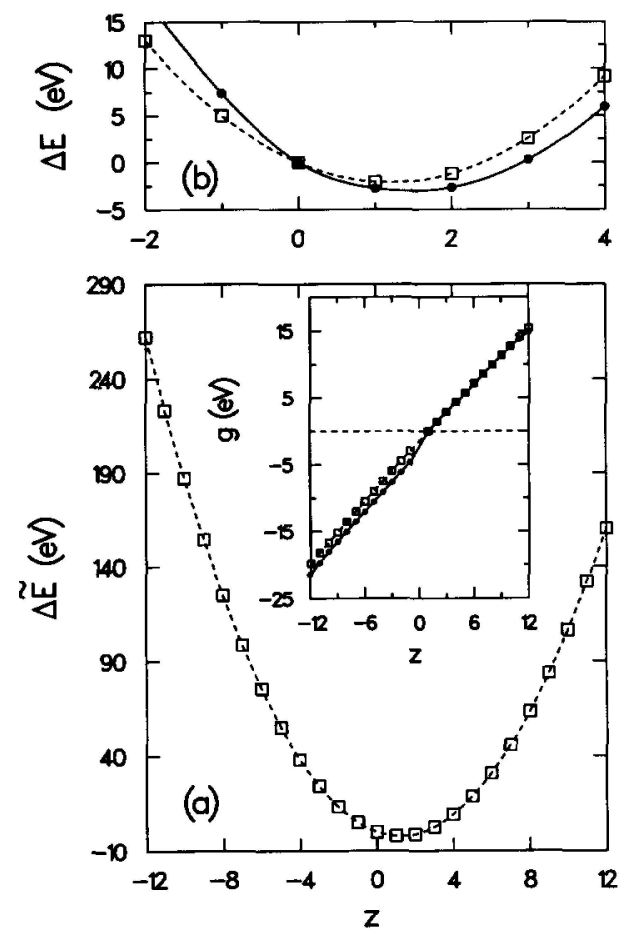

FIG. 4: (a) ETF-LDA total energy differences (appearance energies) $\Delta \widetilde{E}(z)=\widetilde{E}(z)-\widetilde{E}(0)$ as a function of the excess charge $z$ ( $z<0$ corresponds to positive excess charge).

Inset: The ETF function $\widetilde{g}(z)$ (open squares), and the shell-corrected function $g_{\mathrm{sh}}^{\text {ico }}(z)$ (filled circles). For $z \geq 1$ the two functions are almost identical.

(b) magnification of the appearance-energy curves for the region $-2 \leq z \leq 4$. Filled circles: shell-corrected icosahedral values $\left[\Delta E_{\mathrm{sh}}^{\mathrm{ico}}(z)=E_{\mathrm{sh}}^{\mathrm{ico}}(z)-E_{\mathrm{sh}}^{\mathrm{ico}}(0)\right]$. Open squares: ETF-LDA values $[\Delta \widetilde{E}(z)=\widetilde{E}(z)-\widetilde{E}(0)]$

a classical capacitor, corrected for the self-interaction of the excess charge [4, 5]). We remark that regarding the system as a classical conductor, where the excess charge accumulates on the outer surface, yields a value of $C=8.32$ a.u. (that is the outer radius of the jellium shell). Naturally, the ETF calculated value for $C$ is somewhat larger because of the quantal spill-out of the electronic charge density. Indeed, from the slope of $\widetilde{g}(z)$ we determine [46] $C=8.84$ a.u.

A similar plot of the shell-corrected and icosahedrally modified energy differences $\Delta E_{\mathrm{sh}}^{\mathrm{ico}}(z)=E_{\mathrm{sh}}^{\mathrm{ico}}(z)-E_{\mathrm{sh}}^{\mathrm{ico}}(0)$ is shown in Fig. $4 \mathrm{~b}$ (in the range $-2 \leq z \leq 4$, filled circles). The function $g_{\mathrm{sh}}^{\text {ico }}(z)$, defined as in eq. (12) but with the shell-corrected quantities $\left(\Delta E_{\mathrm{sh}}^{\mathrm{ico}}(z)\right.$ and $\left.A_{1}^{\mathrm{ico}}\right)$, is included in the inset to Fig. 4a (filled circles). The shift discernible 
between $g_{\mathrm{sh}}^{\text {ico }}(-1)$ and $g_{\mathrm{sh}}^{\text {ico }}(1)$ is approximately $1.7 \mathrm{eV}$, and originates from the difference of shell effects on the IPs and EAs (see Table I). The effect of shell-closures for $z=-10$ and $z=6$ (which was discussed earlier in the context of Fig. 3b for higher IPs and EAs) is not discernible in the case of $g_{\mathrm{sh}}^{\text {ico }}(z)$ due to the scale of the inset. The segments of the curve $g_{\mathrm{sh}}^{\text {ico }}(z)$ in the inset of Fig. 4a, corresponding to positively $(z<0)$ and negatively $(z>0)$ charged states, are again well approximated by straight lines, whose slope is close to that found for $\widetilde{g}(z)$. Consequently, we may approximate the charging energy, including shell-effects, as follows,

$$
E_{\mathrm{sh}}^{\mathrm{ico}}(x)=E_{\mathrm{sh}}^{\mathrm{ico}}(0)+\frac{x(x-1) e^{2}}{2 C}-A_{1}^{\mathrm{ico}} x
$$

for negatively charged states, and

$$
E_{\mathrm{sh}}^{\mathrm{ico}}(x)=E_{\mathrm{sh}}^{\mathrm{ico}}(0)+\frac{x(x-1) e^{2}}{2 C}+I_{1}^{\mathrm{ico}} x
$$

for positively charged states. Note that without shell-corrections (i.e., ETF) $\widetilde{I}_{1}-\widetilde{A}_{1}=$ $e^{2} / C=27.2 / 8.84 \mathrm{eV} \approx 3.1 \mathrm{eV}$, because of the symmetry of eq. (13) with respect to $z$, while the shell-corrected quantities are related as $I_{1}^{\text {ico }}-A_{1}^{\text {ico }} \approx e^{2} / C+\Delta_{s h}$, where the shell correction is $\Delta_{s h} \approx 1.55 \mathrm{eV}$ (from TABLE I, $\left.I_{1}^{\text {ico }}-A_{1}^{\text {ico }} \approx 4.65 \mathrm{eV}\right)$.

Expression (14) for the negatively charged states can be rearranged as follows (energies in units of $e V$ ),

$$
E_{\mathrm{sh}}^{\mathrm{ico}}(x)-E_{\mathrm{sh}}^{\mathrm{ico}}(0)=-2.99+1.54(x-1.39)^{2},
$$

in close agreement with the all-electron LDA result of Ref. [27].

Equations (14) and (15) can be used to provide simple analytical approximations for the higher IPs and EAs (see the definition in eqs. (10) and (11)). Explicitly written, $A_{x}^{\text {ico }} \equiv$ $E_{\mathrm{sh}}^{\mathrm{ico}}(x-1)-E_{\mathrm{sh}}^{\mathrm{ico}}(x)=A_{1}^{\mathrm{ico}}-(x-1) e^{2} / C$ and $I_{x}^{\mathrm{ico}}=I_{1}^{\mathrm{ico}}+(x-1) e^{2} / C$. Such expressions have been used previously [29] with an assumed value for $C \approx 6.7$ a.u. (i.e., the radius of the $\mathrm{C}_{60}$ molecule, as determined by the distance of carbon nuclei from the center of the molecule), which is appreciably smaller than the value obtained by us $(C=8.84$ a.u., see above) via a microscopic calculation. Consequently, using the above expression with our calculated value for $A_{1}^{\text {ico }}=2.75 \mathrm{eV}$ (see TABLE I), we obtain an approximate value of $A_{2}^{\text {ico }}=-0.35 \mathrm{eV}$ (compared to the microscopically calculated value of $-0.09 \mathrm{eV}$ given in TABLE I, and -0.11 eV obtained by Ref. [27]) — indicating metastability of $\mathrm{C}_{60}^{2-}$ — while 
employing an experimental value for $A_{1}^{\text {ico }}=2.74 \mathrm{eV}$, a value of $A_{2}^{\text {ico }}=0.68 \mathrm{eV}$ was calculated in Ref. [29].

Concerning the cations, our expression (15) with a calculated $I_{1}^{\text {ico }}=7.40 \mathrm{eV}$ (see TABLE I) and $C=8.84 a . u$. yields approximate values $18.5 \mathrm{eV}$ and $31.5 \mathrm{eV}$ for the appearance energies of $\mathrm{C}_{60}^{2+}$ and $\mathrm{C}_{60}^{3+}$ (compared to the microscopic calculated values of $17.71 \mathrm{eV}$ and $30.99 \mathrm{eV}$, respectively, extracted from TABLE I, and $18.6 \mathrm{eV}$ for the former obtained in Ref. [25]). Employing an experimental value for $I_{1}^{\text {ico }}=7.54 \mathrm{eV}$, corresponding values of 19.20 $\mathrm{eV}$ and $34.96 \mathrm{eV}$ were calculated in Ref. [29]. As discussed in Ref. [12], these last values are rather high, and the origin of the discrepancy may be traced to the small value of the capacitance which was used in obtaining these estimates in Ref. [29].

A negative value of the second affinity indicates that $\mathrm{C}_{60}^{2-}$ is unstable against electron autodetachment. In this context, we note that the doubly negatively charged molecule $\mathrm{C}_{60}^{2-}$ has been observed in the gas phase and is believed to be a long-lived metastable species [16, 17]. Indeed, as we discuss in the next section, the small LDA values of $A_{2}^{\text {ico }}$ found by us and by Ref. [27] yield lifetimes which are much longer than those estimated by a pseudopotentiallike Hartree-Fock model calculation [16], where a value of $\sim 1 \mu s$ was estimated.

\section{Lifetimes of metastable anions, $\mathbf{C}_{60}^{x-}$}

The second and higher electron affinities of $\mathrm{C}_{60}$ were found to be negative, which implies that the anions $\mathrm{C}_{60}^{x-}$ with $x \geq 2$ are not stable species, and can lower their energy by emitting an electron. However, unless the number of excess electrons is large enough, the emission of an excess electron involves tunneling through a barrier. Consequently, the moderately charged anionic fullerenes can be described as metastable species possessing a decay lifetime.

To calculate the lifetime for electron autodetachmant, it is necessary to determine the proper potential that the emitted electron sees as it leaves the molecule. The process is analogous to alpha-particle radioactivity of atomic nuclei. The emitted electron will have a final kinetic energy equal to the negative of the corresponding higher EA. We estimate the lifetime of the decay process by using the WKB method, in the spirit of the theory of alphaparticle radioactivity, which has established that the main factor in estimating lifetimes is the relation of the kinetic energy of the emitted particle to the Coulombic tail, and not the details of the many-body problem in the immediate vicinity of the parent nucleus. 
Essential in this approach is the determination of an appropriate single-particle potential that describes the transmission barrier. It is well known that the LDA potential posseses the wrong tail, since it allows for the electron to spuriously interact with itself. A more appropriate potential would be one produced by the Self-Interaction Correction (SIC) method of Ref. [43]. This potential has the correct Coulombic tail, but in the case of the fullerenes presents another drawback, namely Koopman's theorem is not satisfied to an extent adequate for calculating lifetimes. [47] In this context, we note that Koopman's theorem is known to be poorly satisfied for the case of fullerenes even in Hartree-Fock calculations [30]. Therefore, the HOMO corresponding to the emitted electron, calculated as described above, cannot be used in the WKB tunneling calculation.

Since the final energy of the ejected electron equals the negative of the value of the electron affinity, we seek a potential that, together with the icosahedral perturbation, yields a HOMO level in $\mathrm{C}_{60}^{x-}$ with energy $-A_{x}^{\text {ico }}$. We construct this potential through a selfinteraction correction to the LDA potential as follows,

$$
V_{\mathrm{WKB}}=V_{\mathrm{LDA}}[\widetilde{\rho}]-V_{H}\left[\frac{\widetilde{\rho}}{N_{e}}\right]-V_{\mathrm{xc}}\left[\xi \frac{\widetilde{\rho}}{N_{e}}\right]
$$

where the parameter $\xi$ is adjusted so that the $\mathrm{HOMO}$ level of $\mathrm{C}_{60}^{x-}$ equals $-A_{x}^{\text {ico }}$. In the above expression, the second term on the rhs is an average self-interaction Hartree correction which ensures a proper long-range behavior of the potential (i.e., correct Coulomb tail), and the third term is a correction to the short-range exchange-correlation.

For the cases of $\mathrm{C}_{60}^{2-}$ and $\mathrm{C}_{60}^{3-}$ such potentials are plotted in Fig. 5. We observe that they have the correct Coulombic tail, namely a tail corresponding to one electron for $\mathrm{C}_{60}^{2-}$ and to two electrons for $\mathrm{C}_{60}^{3-}$. The actual barrier, however, through which the electron tunnels is the sum of the Coulombic barrier plus the contribution of the centrifugal barrier. As seen from Fig. 5, the latter is significant, since the HOMO in the fullerenes possesses a rather high angular momentum $(l=5)$, while being confined in a small volume.

Using the WKB approximation [44], we estimate for $\mathrm{C}_{60}^{2-}$ a macroscopic half-life of $\sim$ $4 \times 10^{7}$ years, while for $\mathrm{C}_{60}^{3-}$ we estimate a very short half-life of $2.4 \times 10^{-12} \mathrm{~s}$. Both these estimates are in correspondence with observations. Indeed, $\mathrm{C}_{60}^{3-}$ has not been observed as a free molecule, while the free $\mathrm{C}_{60}^{2-}$ has been observed to be long lived [16, 17] and was detected even 5 min after its production through laser vaporization [17].

We note that the WKB lifetimes calculated for tunneling through Coulombic barriers are 


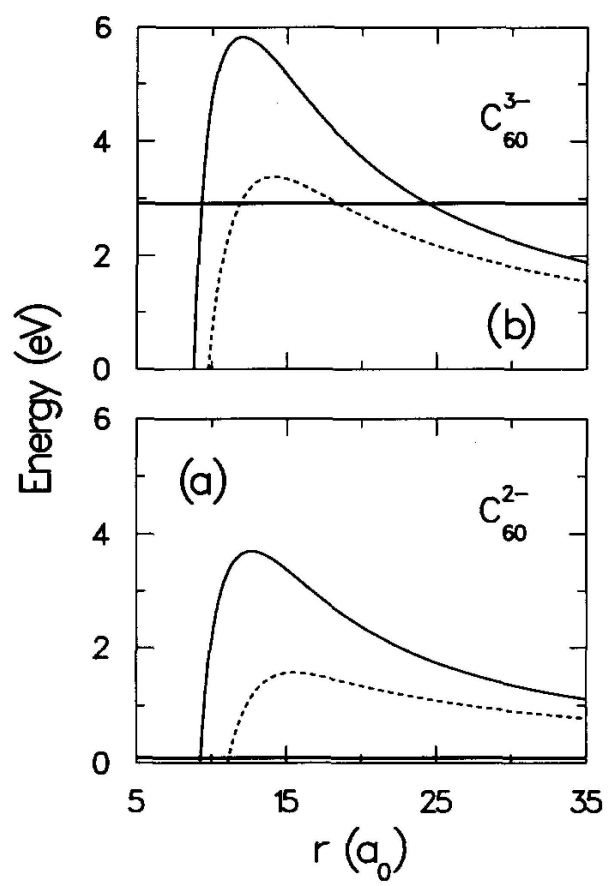

FIG. 5: WKB effective barriers used to estimate lifetimes for $\mathrm{C}_{60}^{2-}$ (a) and $\mathrm{C}_{60}^{3-}$ (b). Dashed lines correspond to barriers due solely to Coulombic repulsion and solid lines to total barriers after adding the centrifugal components. The thick horizontal solid lines correspond to the negative of the associated electron affinities $A_{2}^{\text {ico }}$ (a) and $A_{3}^{\text {ico }}$ (b). In the case of $\mathrm{C}_{60}^{2-}$ [panel (a)], the horizontal solid line at $-A_{2}^{\text {ico }}=0.09 \mathrm{eV}$ crosses the total barrier at an inside point $R_{1}=9.3$ a.u. and again at a distance very far from the center of the fullerene molecule, namely at an outer point $R_{2}=-e^{2} / A_{2}^{\text {ico }}=27.2 / 0.09$ a.u. $=302.2$ a.u. This large value of $R_{2}$, combined with the large centrifugal barrier, yields a macroscopic lifetime for the metastable $\mathrm{C}_{60}^{2-}$ (see text for details).

very sensitive to the final energy of the emitted particle and can vary by many orders of magnitude as a result of small changes in this energy, a feature well known from the alpha radioctivity of nuclei [44]. Since the second electron affinity of $\mathrm{C}_{60}$ is small, effects due to geometrical relaxation and spin polarization can influence its value and, consequently, the estimated lifetime. Nevertheless, as shown in Ref. [27], inclusion of such corrections yields again a negative second affinity, [48] but of somewhat smaller magnitude, resulting in an even longer lifetime. Furthermore, as discussed in Ref. [28], the stabilization effect of the Jahn-Teller relaxation for the singly-charged ion is only of the order of $0.03-0.05 \mathrm{eV}$. Since this effect is expected to be largest for singly-charged species, $\mathrm{C}_{60}^{2-}$ is not expected to be 
influenced by it [27].

On the other hand, generalized exchange-correlation functionals with gradient corrections yield slightly larger values for the second electron affinity. For example, using exchangecorrelation gradient corrections, Ref. [27] found $A_{2}^{\text {ico }}=-0.3 \mathrm{eV}$, which is higher (in absolute magnitude) than the value obtained without such corrections. This value of $-0.3 \mathrm{eV}$ leads to a much smaller lifetime than the several million of years that correspond to the value of $-0.09 \mathrm{eV}$ calculated by us. Indeed, using the barrier displayed in Fig. 5a, we estimate a lifetime for $\mathrm{C}_{60}^{2-}$ of approx. $0.37 \mathrm{~s}$, when $A_{2}^{\text {ico }}=-0.3 \mathrm{eV}$. We stress, however, that even this lower-limit value still corresponds to macroscopic times and is 5 orders of magnitude larger than the estimate of Ref. [16], which found a lifetime of $1 \mu \mathrm{s}$ for $A_{2}^{\text {ico }}=-0.3 \mathrm{eV}$, since it omitted the large centrifugal barrier. Indeed, when we omit the centrifugal barrier, we find a lifetime estimate of $1.4 \mu \mathrm{s}$, when $A_{2}^{\text {ico }}=-0.3 \mathrm{eV}$.

\section{SUMMARY}

We developed a new LDA method for investigation of complex carbon clusters, and, as an illustration, applied it to multiply charged fullerenes $\mathrm{C}_{60}^{x \pm}$. The main elements of this method are: (i) Use of a stabilized jellium (structureless pseudopotential) approximation [20] for the ionic background, instead of the standard jellium approximation; (ii) Use of a recently introduced, shell-correction method [4, 5]; (iii) Inclusion of the effect of the icosahedral symmetry via a perturbative treatment.

For the neutral $\mathrm{C}_{60}$ molecule, the results obtained by our method are in good agreement with experimental observations, as well as with previous self-consistent electronic structure calculations [24, 25, 26].

For the multiply charged fullerenes, charging energies were microscopically calculated for up to $x=12$ excess charges. A semiclassical interpretation of these results was developed, which viewed the fullerenes as Coulomb islands [2, 3] possessing a classical capacitance. A value of 8.84 a.u. was found for this capacitance, in contrast to the much smaller value of 6.7 a.u. inferred previously from the particle-on-a-sphere model [29]. The calculated values for the first ionization potential and the first electron affinity agree well with the experimental ones. For the second and third ionization potentials, there exist substantial discrepancies in the experimental measurements [12]. Our calculations, reflecting the more accurate value of 
the capacitance of $\mathrm{C}_{60}$, support the results from charge transfer bracketing experiments [11] and from direct ionization experiments by electron impact [12].

Employing the analogies with the case of alpha-particle radioactivity in nuclei, we found that the doubly charged negative ion is a very long-lived metastable species, in agreement with observations [16, 17]. It decays through spontaneous electron emission, and its macroscopic lifetime is the result of a superposition of a Coulombic barrier and a large centrifugal barrier, through which the emitted electron tunnels.

\section{Acknowlegdments}

This research is supported by the U.S. Department of Energy, Grant No. AG0586ER45234.

[1] C. Coulomb, Mémoires de l'Académie, (1786) p. 67ff; ibid. (1787) p. 452; M. Faraday, Experimental research in electricity, (1839) (Dover, New York, 1965), Vol. I, p. 360ff.

[2] M.A. Kastner, Artificial atoms, in Physics Today, vol. 46 (1993) 24.

[3] Single Charge Tunneling, eds. H. Grabert and M.H. Devoret, (Plenum, New York, 1992).

[4] C. Yannouleas and U. Landman, Chem. Phys. Lett. 210 (1993) 437.

[5] C. Yannouleas and U. Landman, Phys. Rev. B 48 (1993) 8376.

[6] For experimental studies of charged cationic clusters, see C. Bréchignac, Ph. Cahuzac, F. Carlier, and M. de Frutos, Phys. Rev. Lett. 64 (1990) 2893; C. Bréchignac, Ph. Cahuzac, F. Carlier, J. Leygnier, and A. Sarfati, Phys. Rev. B 44 (1991) 11 386; T.P. Martin et al. Chem. Phys. Lett. 196 (1992) 113, and references therein.

[7] For a recent review of singly charged anionic metal clusters, see J. G. Eaton, L. H. Kidder, H. W. Sarkas, K. M. McHugh, and K. H. Bowen, in Nuclear Physics Concepts in the Study of Atomic Cluster Physics, R. Schmidt et al Eds., (Springer, Berlin, 1992), Lecture Notes in Physics Vol. 404, p. 291.

[8] J. V. Coe, G. H. Lee, J. G. Eaton, S. T. Arnold, H. W. Sarkas, K. H. Bowen, C. Ludewigt, H. Haberland, and D. R. Worsnop, J. Chem. Phys. 92 (1990) 3980.

[9] R. N. Barnett, U. Landman, C. L. Cleveland, and J. Jortner, J. Chem. Phys. 88 (1988) 4421; 
ibid. 88 (1988) 4429; H.-P. Kaukonen, R. N. Barnett, and U. Landman, J. Chem. Phys. 97 (1992) 1365.

[10] D. Scharf, J. Jortner, and U. Landman, J. Chem. Phys. 87 (1987) 2716.

[11] S.W. McElvany, M.M. Ross, and J.H. Callahan, Mater. Res. Soc. Symp. Proc. 206 (1991) 697; Acc. Chem. Res., 25 (1992) 162.

[12] M. Sai Baba, T.S. Lakshmi Narasimhan, R. Balasubramanian, and C.K. Mathews, Int. J. Mass Spectrom. Ion Processes 125 (1993) R1.

[13] R.K. Yoo, B. Ruscic, and J. Berkowitz, J. Chem. Phys. 96 (1992) 911.

[14] C.W. Walter, Y.K. Bae, D.C. Lorents, and J.R. Peterson, Chem. Phys. Lett. 195 (1992) 543.

[15] H. Steger, J. de Vries, B. Kamke, W. Kamke, and T. Drewello, Chem. Phys. Lett. 194 (1992) 452.

[16] R.L. Hettich, R.N. Compton, and R.H. Ritchie, Phys. Rev. Lett. 67 (1991) 1242.

[17] P.A. Limbach, L. Schweikhard, K.A. Cowen, M.T. McDermott, A.G. Marshall, and J.V. Coe, J. Am. Chem. Soc. 113 (1991) 6795.

[18] S.N. Schauer, P. Williams, and R.N. Compton, Phys. Rev. Lett. 65 (1990) 625.

[19] W. Kohn and L. J. Sham, Phys. Rev. 140 (1965) A1133.

[20] J.P. Perdew, H.Q. Tran, and E.D. Smith, Phys. Rev. B 42 (1990) 11627.

[21] H. Kuhn, J. Chem. Phys. 17 (1949) 1198.

[22] G.A. Gallup, Chem. Phys. Lett. 187 (1991) 187.

[23] M.R. Savina, L.L. Lohr, and A.H. Francis, Chem. Phys. Lett. 205 (1993) 200.

[24] N. Troullier and J.L. Martins, Phys. Rev. B 46 (1992) 1754; J.L. Martins, N. Troullier, and J.H. Weaver, Chem. Phys. Lett. 180 (1991) 457.

[25] Arne Rosén and Bo Wästberg, J. Chem. Phys. 90 (1989) 2525; Bo Wästberg and Arne Rosén, Physica Scripta 44 (1991) 276.

[26] L. Ye and A.J. Freeman, Chem. Phys. 160 (1992) 415.

[27] M.R. Pederson and A.A. Quong, Phys. Rev. B 46 (1992) 13584.

[28] V. de Coulon, J.L. Martins, and F. Reuse, Phys. Rev. B 45 (1992) 13671.

[29] Y. Wang, D. Tománek, G.F. Bertsch, and R.S. Ruoff, Phys. Rev. B 47 (1993) 6711.

[30] J. Cioslowski and K. Raghavachari, J. Chem. Phys. 98 (1993) 8734.

[31] J. Hutter and H.P. Lüthi, Int. J. Quantum Chem. 46 (1993) 81.

[32] J. Kohanoff, W. Andreoni, and M. Parrinello, Chem. Phys. Lett. 198 (1992) 472. 
[33] K. Yabana and G.F. Bertsch, Jellium model for $C_{60}$, preprint (1992).

[34] N. Van Giai and E. Lipparini, Z. Phys. D 27 (1993) 193.

[35] M.J. Puska and R.M. Nieminen, Phys. Rev. A 47 (1993) 1181.

[36] C. Yannouleas, E. Vigezzi, and R.A. Broglia, Phys. Rev. B47 (1993) 9849; C. Yannouleas, Chem. Phys. Lett. 193 (1992) 587; C. Yannouleas, P. Jena, and S. N. Khanna, Phys. Rev. B 46 (1992) 9751; C. Yannouleas and R. A. Broglia, Phys. Rev. A 44 (1991) 5793; C. Yannouleas, R.A. Broglia, and M. Brack, Phys. Rev. Lett. 63 (1989) 255.

[37] B. Grammaticos, Z. Phys. A 305 (1982) 257.

[38] J. Harris, Phys. Rev. B 31 (1985) 1770.

[39] R.C. Haddon, L.E. Brus, and K. Raghavachari, Chem. Phys. Lett. 125 (1986) 459.

[40] M. Gerloch and R.C. Slade, Ligand field parameters (Cambridge Univ. Press, London, 1973).

[41] S.H. Yang, C.L. Pettiette, J. Conceico, O. Chesnovsky, and R.E. Smalley, Chem. Phys. Lett. 139 (1987) 233.

[42] I.V. Hertel, H. Steger, J. de Vries, B. Weisser, C. Menzel, B. Kamke, and W. Kamke, Phys. Rev. Lett. 68 (1992) 784.

[43] J. P. Perdew and A. Zunger, Phys. Rev. B 23 (1981) 5048.

[44] A. I. Baz', Y. B. Zel'dovich, and A. M. Perelomov, Scattering, reactions, and decay in nonrelativistic quantum mechanics (Israel Program for Scientific Translations Ltd., Jerusalem, 1969).

[45] Other aspects differentiating our study from previous ones are: Consideration of the icosahedral perturbation, unlike Refs. 34, 35]; consideration of 240 active electrons (as is the case with ab initio LDA pseudopotential claculations, see i.e., Ref. [24]), unlike Ref. [33] which considered 360 active electrons, Ref. [34] which considered 60 active electrons, and Ref. [35] which considered 250 active electrons.

[46] Due to the changing spill-out with excess charge $z$, the capacitance should be written as $C+\delta(z)$. For our purposes here the small correction $\delta(z)$ can be neglected.

[47] For certain systems, such as for example sodium clusters, an orbitally-averaged-like SIC treatment yielded highest-occupied-molecular-orbital (HOMO) energies for anions in adequate agreement with the calculated electron affinities (see, Refs. [4, 5]).

[48] The sign conventions in Ref. [27] are the opposite of ours. 